\title{
Loss of Purkinje Cells is Associated with Demyelination in Multiple Sclerosis
}

\author{
Fabrizio Giuliani, Ingrid Catz, Edward Johnson, Lothar Resch, Kenneth Warren
}

Can. J. Neurol. Sci. 2011; 38: 529-531

Multiple sclerosis (MS) is an inflammatory demyelinating disease of the central nervous system (CNS) previously thought to involve mainly the white matter. In the past 10-15 years, pathology and MRI studies have shown that in addition to white matter lesions, there is significant involvement of the gray matter as well as significant axonal and neuronal loss ${ }^{1}$. Involvement of the cerebellar cortex is not commonly described in MS, although in a recent report Kutzelnigg et al have described extensive demyelination in the cerebellar cortex which was associated with relative axonal and neuronal preservation ${ }^{2}$.

Here, we report Purkinje cell loss in two cases of multiple sclerosis, one of the classical Charcot variant and the other of the acute Marburg variant.

At age 30, a male patient developed right hemianesthesia and paresthesiae which resolved within a few weeks. Five years later he complained of numbness of both hands and decreased vision in the left eye. At this time, visual evoked responses were abnormal and MRI of the brain was suggestive of MS. Later during the same year, the patient complained of balance problems that made it difficult to jump on and off trains at his vocation as train engineer. By the end of the second year the patient had spastic paraparesis with extensor plantar responses. His gait deteriorated and he developed spastic quadriparesis. Seven years after the onset the patient was in a wheelchair and had significant difficulty standing up or transferring in and out of bed or on and off the toilet. A repeat MRI showed extensive white matter changes in the cerebral hemispheres but no disease in the cerebellum although the right middle cerebellar peduncle was affected. After ten years of MS, the patient had developed pendular nystagmus in both eyes, tremor and dysarthria, severe contractures, multiple and extensive decubitus ulcers. He died with aspiration pneumonia at age 49 .

A post mortem examination of the nervous system confirmed the diagnosis of MS affecting the cerebral hemispheres and the corpus callosum, the thalamus and hypothalamus, the brain stem, the spinal cord and the cerebellum. In the cerebellum, Purkinje cells loss was associated with focal demyelination in adjacent folia (Figure 1).

A 17-year-old girl suddenly lost the vision in her right eye. During the subsequent two weeks she developed numbness of her left arm and leg. Within three months she developed bilateral visual loss, nausea and vertigo, and profound loss of hearing in both ears. Asymmetrical weakness, worse on the right side, and urinary retention quickly developed. Nystagmus of high amplitude was present on conjugate gaze to the left and to the right and hearing was absent in the left ear and reduced in the right. Asymmetrical quadriparesis was present. Bilateral lower limb ataxia was worse on the left side. Cerebral spinal fluid

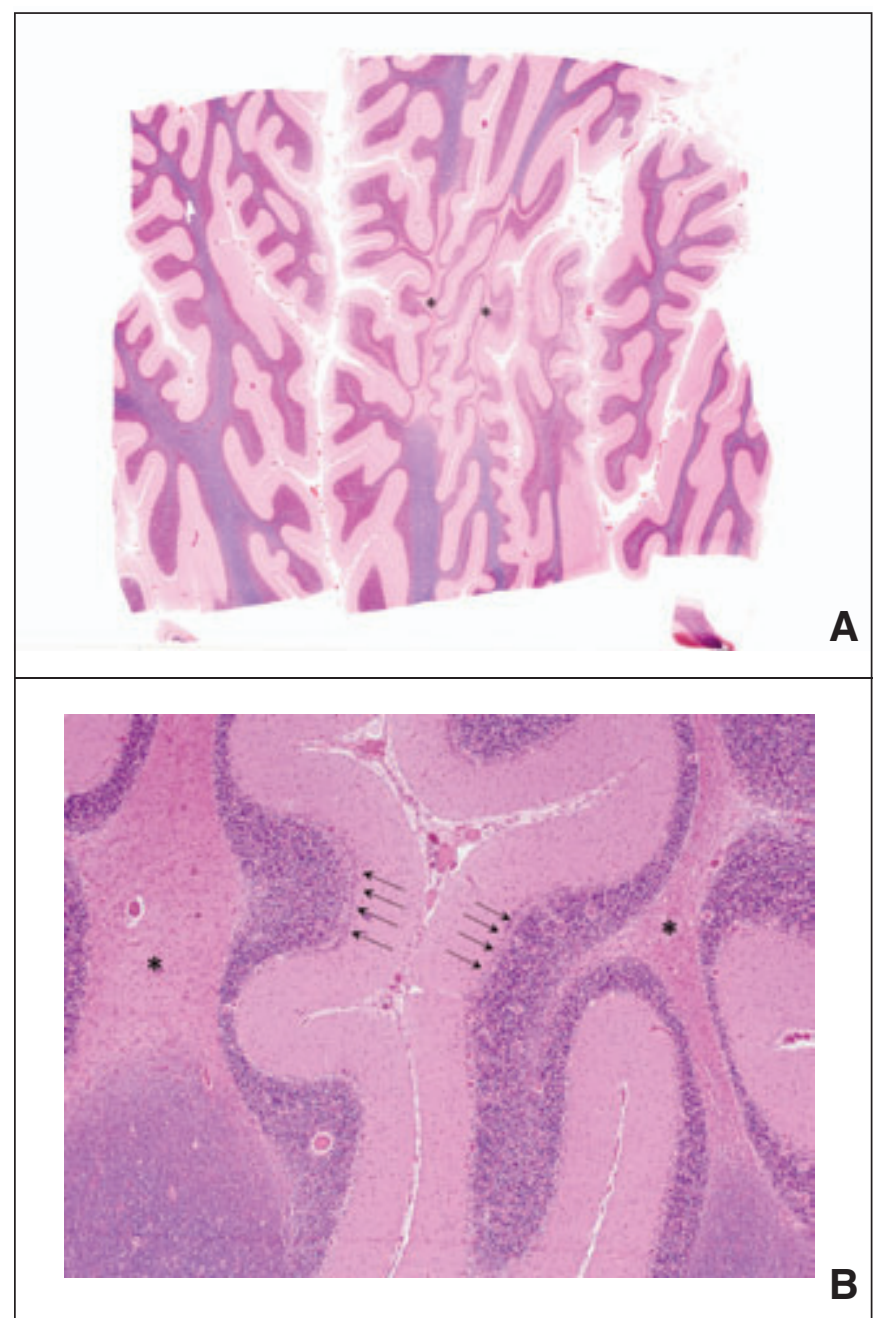

Figure 1: (A) Normal folia on the left and MS plaques on the right (*). There is atrophy of the portion of the folium affected by the plaque. Luxol Fast Blue/Hematoxylin Eosin stain (LFB/HE) 1x. (B) High magnification of MS plaque (*). Purkinje cell loss replaced by Bergman gliosis (arrows). LFB/HE 100x.

From the Department of Medicine (Neurology) (FG, IC, KW), Laboratory Medicine and Pathology (Neuropathology) (EJ, LR), University of Alberta, Edmonton, Alberta, Canada.

Received September 22, 2010. Final Revisions Submitted December 1, 2010. Correspondence to: Fabrizio Giuliani, Division of Neurology - Department of Medicine, University of Alberta, 9-101 Clinical Sciences Building - University of Alberta, Edmonton, Alberta, T6G 2G3, Canada. 

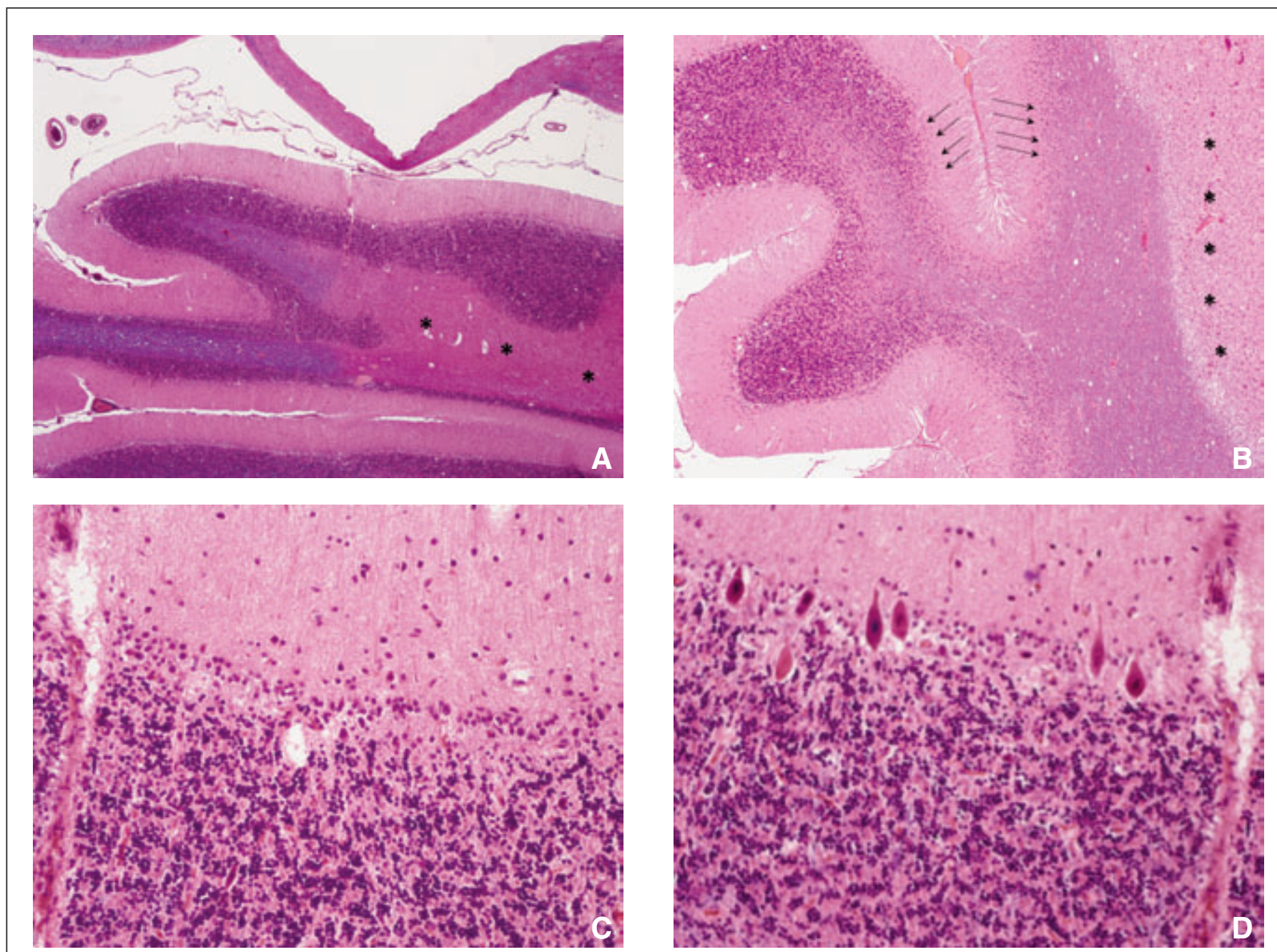

Figure 2: (A) A large diffuse plaque (*) extending into the cerebellar folium. LFB/HE 1x. (B) An acute active cellular MS plaque (*) and loss of nearby Purkinje cells (arrows). LFB/HE. (C) A higher magnification showing loss of Purkinje cells and reactive Bergmann astrogliosis. LFB/HE 100x. (D) Higher magnification showing occasionally plaque-associated hypereosinophyllic pyknotic Purkinje cells. LFB/HE 100x.

(CSF) examination on three separate occasions illustrated 38, 27 and 1 white blood cells; protein of 95,69 and $88 \mathrm{~g} / 1$ and positive oligoclonal banding. Five months from the disease onset, the patient entered into a partial remission. One month later she again rapidly deteriorated with severe dysarthria and dysphagia, conjugate gaze palsy to the left, eye deviation to the right and with paralyzed lower extremities. Her legs were completely paralyzed. Within four weeks she was in a locked-in syndrome: she could blink her eyes on command, but was unable to move them vertically or horizontally. She developed increasing respiratory difficulties and died with pneumonia 45 weeks after the onset.

Post mortem examination showed large plaques of demyelination with numerous infiltrating inflammatory cells throughout the central nervous system. Within the cerebellum, the corpus medullare as well as primary, secondary and tertiary folia showed diffuse demyelination which was associated with diffuse Purkinje cell loss (Figure 2).
Cerebellar involvement in MS is a frequent clinical finding, it affects as much as one third of the patient population and is a significant contributor to disability ${ }^{2,3}$. In our two cases, Purkinje cell loss was associated with demyelinating plaques in adjacent folia: focal demyelination was associated with focal Purkinje cell loss in the Charcot variant and diffuse demyelination was associated with diffuse Purkinje cell loss in the Marburg variant. Although Purkinje cells are sensitive to hypoxia-mediated cell death, in the cases presented above, Purkinje cell loss was only associated with demyelinating plaques in the adjacent white matter. We did not observe any reduction of Purkinje cells in areas adjacent to 'normal appearing' white matter. In the patient with Marburg MS, diffuse inflammation and demyelination may have accounted for diffuse Purkinje cell loss. Inflammation and demyelination could induce transection of Purkinje axons, and of mossy and climbing fibers projecting to Purkinje cells with subsequent retrograde and transneuronal degeneration ${ }^{4,5}$. One of 
us $^{5}$ has previously shown that human cortical neurons are susceptible to inflammatory-mediated cytotoxicity. Indeed, additional neuropathological studies to correlate demyelination with neuronal loss in MS would be beneficial.

A number of limitations to our work warrant further discussion. Firstly, this is a report of only two cases, one with the Charcot variant of chronic progressing MS and the other with the Marburg variant of acute disease. Secondly, although Purkinje cell loss was qualitatively significant in both cases, no quantification has been done to compare to control subjects. Thirdly, because of the limited number of cases, it was difficult to comment on the functional significance of our findings (e.g. correlation of Purkinje cell number with disability).

Further work is required to clarify the mechanisms of Purkinje cell loss in MS and its clinical significance in the different subtypes of the disease.

\section{REFERENCES}

1. Rudick RA, Trapp BD. Gray-matter injury in multiple sclerosis. $\mathbf{N}$ Engl J Med. 2009;361:1505-6.

2. Kutzelnigg A, Faber-Rod JC, Bauer J, et al. Widespread demyelination in the cerebellar cortex of multiple sclerosis. Brain Pathol. 2007;17:38-44.

3. Alusi SH, Worthington J, Glickman S, et al. A study of tremor in multiple sclerosis. Brain. 2001;124:720-30.

4. Trapp BD, Nave KA. Multiple Sclerosis' an immune or neurodegenerative disorder? Ann Rev Neurosci. 2006;31:247-69.

5. Giuliani F, Goodyer CG, Antel JP, et al. Vulnerability of human neurons to T cell-mediated cytotoxicity. J Immunol. 2003;171: 368-79. 